\title{
Interactive neonatal gastrointestinal magnetic resonance imaging using fruit juice as an oral contrast media
}

\author{
Owen J Arthurs ${ }^{1,2,3^{*}}$, Martin J Graves ${ }^{1}$, Andrea D Edwards ${ }^{1}$, Ilse Joubert ${ }^{1}$, Pat AK Set ${ }^{1}$ and David J Lomas ${ }^{1}$
}

\begin{abstract}
Background: The objective was to evaluate the use of fruit juice with an interactive inversion recovery (IR) MR pulse sequence to visualise the gastrointestinal tract.

Methods: We investigated the relaxation properties of 12 different natural fruit juices in vitro, to identify which could be used as oral contrast. We then describe our initial experience using an interactive MR pulse sequence to allow optimal visualisation after administering pineapple juice orally, and suppressing pre-existing bowel fluid contents, with variable TI in three adult and one child volunteer.

Results: Pineapple juice (PJ) had both the shortest $T_{1}(243 \mathrm{~ms})$ and shortest $\mathrm{T}_{2}(48 \mathrm{~ms})$ of the fruit juices tested. Optimal signal differentiation between pre-existing bowel contents and oral PJ administration was obtained with Tls of between 900 and 1100 ms.

Conclusion: The use of an inversion recovery preparation allowed long $T_{1}$ pre-existing bowel contents to be suppressed whilst the short $T_{1}$ of fruit juice acts as a positive contrast medium. Pineapple juice could be used as oral contrast agent for neonatal gastrointestinal magnetic resonance imaging.
\end{abstract}

Keywords: Neonatal, MRI, Bowel, Fruit juice

\section{Background}

Congenital abnormalities of gut rotation in neonates and infants are usually investigated by the upper gastrointestinal contrast study (UGI), using traditional X-ray fluoroscopy techniques. Malrotation is typically excluded on the basis of confidently identifying a normally positioned duodeno-jejunal (DJ) flexure, usually on the first pass of radio-opaque oral contrast medium through the duodenum at UGI [1]. However, these procedures require skilled interpretation, carry a significant radiation burden to the child, and may not always be diagnostic [2]. Optimal imaging of the neonatal GI tract is currently under review, with ongoing debate in the literature as to whether ultrasound or cross sectional imaging (such as CT) could replace the UGI series $[3,4]$, although both have limitations,

\footnotetext{
* Correspondence: owen.arthurs@gosh.nhs.uk

'Department of Radiology, Cambridge University Hospitals NHS Foundation

Trust, Cambridge CB2 OQQ, UK

2Department of Radiology, Great Ormond Street Hospital for Children NHS

Foundation Trust, London, UK

Full list of author information is available at the end of the article
}

including ionising radiation doses in CT higher than that of UGI, and a significant false negative rate just as with UGI.

Recent advances in magnetic resonance imaging (MRI), particularly the development of interactive multi-contrast pulse sequences, may now provide an alternative imaging technique, thus avoiding ionising radiation. Interactive MRI allows real-time control over image plane location as well as a number of image acquisition parameters, including the development of an IR based Single Shot Fast Spin Echo (IR-SSFSE) sequence $[5,6]$. This could be useful in the setting of possible gut malrotation, just as interactive MRI has been used to visualise vesicoureteric reflux in the neonatal urinary tract [7]. However, in order to closely replicate the UGI series using MRI, an easily visualised contrast medium is needed.

The ideal medium should be easily tolerated, provide high positive signal enhancement, and help to distinguish the DJ flexure from pre-existing bowel contents in adjacent small bowel (likely to be a combination of milk or 
water-based material in neonates). There are several practical limitations in neonatal gut MRI, particularly regarding the choice of oral contrast agent. This is largely determined by their signal properties, the imaging modality used, the degree of gut distension required, and therefore the minimum volume of contrast agent required. Traditionally, barium sulphate is used in conventional radiographic gastrointestinal studies, but where there is a risk of spillage into the respiratory tract or perforation (particularly in paediatric studies) it has been replaced by non-ionic, low osmolality compounds (e.g. Iopamidol; Gastromiro, Bracco, High Wycombe, UK). High density barium has been used as a negative oral contrast agent for MR imaging of the gut [8], but typically requires high volumes of contrast medium to be ingested, which is not practical for neonates and small children.

Administering water (high contrast on $\mathrm{T}_{2} \mathrm{w}$ images) may not allow confident identification of the DJ flexure separately from the pre-existing fluid in adjacent jejunal loops, a key diagnostic factor for the UGI series. Currently, there are no gadolinium-based contrast agents licensed for oral use in children, but several commercially available (i.e. commodity) fruit juices have been used as both positive and negative oral contrast agents. For example, the paramagnetic properties of fruit juices such as concentrated pineapple or blueberry juice have been used to suppress the signal from bowel fluid in paediatric magnetic resonance cholangio-pancreatography (MRCP) studies which employ heavily $\mathrm{T}_{2}$-weighted imaging $[9,10]$. As well as their $T_{2}$ effects, the $T_{1}$ shortening properties of these juices may also make them suitable positive contrast agents. However, they cannot be administered in high concentrations to neonates, due to the inability of the immature gut to deal with complex carbohydrate loads (of greater than 10\%; [11]).

Administering an oral contrast medium with unknown positive signal properties, to an infant with unknown signal properties of small bowel content provides a wide range of variables. In this study, we first investigated the relaxation properties of several diluted fruit juices in vitro, to identify which could be used as oral contrast. We then describe our initial experience using an interactive $M R$ pulse sequence, whereby real-time control over imaging parameters such as IR pulse timing could allow optimal contrast medium visualisation by suppressing pre-existing bowel fluid contents.

\section{Methods}

We optimised the interactive IR-SSFSE sequence as part of an ethically approved study into optimising gut imaging (Cambridgeshire 3 Research Ethics Committee formal approval 08/H0306/7). Three adult volunteers and one child participated, who were nil-by-mouth for 6 hours and
3 hours respectively prior to the MR examination. Full informed written consent was obtained from each participant and the child's parent. No sedation or anaesthesia was used. Ethical approval was not required for in-vitro studies.

\section{Contrast agent testing}

12 different contrast media were prepared, as follows: Tap water, standard infant formula milk (SMA Gold, SMA nutrition, Berkshire, UK), Iopamidol (Gastromiro, Bracco, High Wycombe, UK), and the following 9 commercially available (standard supermarket stock) fruit juices: orange juice, two examples of pineapple juice (one from juice; PJ, and one reconstituted from concentrate, PJ2), apple \& beetroot juice, prune juice, blackcurrant juice, blueberry juice, raspberry juice, and blackberry juice. Three of these products (PJ2, prune, and blueberry juice) required further dilution to achieve a carbohydrate concentration of $<10 \%$, all other media were imaged undiluted (see Table 1). Estimated nutritional and paramagnetic agent contents of each juice are given (Table 2).

Each proposed contrast agent was placed within a multicompartment phantom positioned inside an 8 channel brain array coil, and images acquired on a standard $1.5 \mathrm{~T}$ whole-body MR system (Signa HDx; GE Healthcare, Waukesha, WI; Figure 1).

The $\mathrm{T}_{1}$ values were measured using a $2 \mathrm{D}$ inversion recovery (IR) prepared fast radiofrequency (RF) spoiled gradient echo (FSPGR) sequence with variable inversion times (TI) at nine different TIs $(40,80,160,320,640 \mathrm{~ms}$, and $1.28,2.56,5.12,10.24 \mathrm{sec})$. Other imaging parameters

\begin{tabular}{|c|c|c|c|c|}
\hline & Solution & $\mathrm{COH}$ conc (per $100 \mathrm{ml})$ & $\mathrm{T}_{1}$ (ms) & $\mathrm{T}_{2}$ (ms) \\
\hline 1 & Water & 0 & 2831 & 1950 \\
\hline 2 & Milk & $7.3 \%$ & 930 & 148 \\
\hline 3 & lopamidol & 0 & 810 & 165 \\
\hline 4 & Orange & $9.0 \%$ & 1796 & 440 \\
\hline 5 & PJ & $10.0 \%(12.7 \% *)$ & 243 & 48 \\
\hline 6 & PJ2 & $8.6 \%$ & 258 & 53 \\
\hline 7 & Apple/beetroot & $9.6 \%$ & 1679 & 518 \\
\hline 8 & Prune & $10.0 \%(16.8 \% *)$ & 1245 & 324 \\
\hline 9 & Blackcurrant & $1.0 \%$ & 2803 & 1480 \\
\hline 10 & Blueberry & $10.0 \%(10.5 \% *)$ & 573 & 80 \\
\hline 11 & Raspberry & $0.3 \%$ & 1055 & 146 \\
\hline 12 & Blackberry & 0 & 831 & 103 \\
\hline
\end{tabular}

$\mathrm{COH}$ concentration was provided from manufacturers packaging, and not tested directly.

3 juices $(*)$ had too high a $\mathrm{COH}$ concentration, and so were diluted down to $10 \%$ prior to testing: the original concentrations are given in parentheses. 
Table 2 Contents of each juice tested as provided by manufacturer

\begin{tabular}{|c|c|c|c|c|c|c|c|c|c|c|}
\hline & Solution & Manufacturer & Description as purchased & $\begin{array}{l}\text { Energy } \\
\text { (kcal) }\end{array}$ & $\begin{array}{l}\text { Protein } \\
\text { (g) }\end{array}$ & $\begin{array}{l}\mathrm{COH} \\
\text { (g) }\end{array}$ & $\begin{array}{l}\text { Fat } \\
\text { (g) }\end{array}$ & $\begin{array}{l}\text { Iron } \\
\text { (mg) }\end{array}$ & $\begin{array}{l}\text { Manganese } \\
\text { (mg) }\end{array}$ & $\begin{array}{l}\text { Copper } \\
\text { (mg) }\end{array}$ \\
\hline 2 & Milk & SMA Gold & SMA Gold & 67 & 1.9 & 7.3 & 3.3 & 0.64 & 0.09 & 0.06 \\
\hline 4 & Orange & Sainsburys & Pure orange juice (conc) & 47 & 0.5 & 9.0 & trace & 0.12 & 0.04 & 0.02 \\
\hline 5 & PJ & Sainsburys & Pure pineapple juice (conc) & 54 & 0.1 & 12.7 & 0.0 & 0.29 & 0.92 & 0.11 \\
\hline 6 & PJ2 & Marks \& Spencers & 99\% pineapple juice & 35 & 0.5 & 8.6 & 0.1 & 0.29 & 0.92 & 0.11 \\
\hline 7 & Apple/beetroot & Marks \& Spencers & Pressed apple \& beetroot & 40 & 0.3 & 9.6 & 0.1 & 0.12 & 0.07 & 0.01 \\
\hline 8 & Prune & Tesco & Pure prune juice & 75 & 0.7 & 16.8 & 0.1 & 1.18 & 0.15 & 0.07 \\
\hline 9 & Blackcurrant & Disney & Blackcurrant with sweetener & 2 & trace & 1.0 & trace & 1.54 & 0.26 & 0.08 \\
\hline 10 & Blueberry & Sainsburys & Blueberry juice drink (conc) & 44 & 0.1 & 10.5 & trace & 0.28 & 0.33 & 0.06 \\
\hline 11 & Raspberry & Sainsburys & Juice & 30 & 0.4 & 0.3 & 0 & 0.35 & 0.33 & 0.04 \\
\hline 12 & Blackberry & Twinings tea & $\begin{array}{l}2 \% \text { blackberry flavouring, } \\
10 \% \text { blackberry leaves }\end{array}$ & 0 & 0 & 0 & 0 & 0.31 & 0.32 & 0.08 \\
\hline
\end{tabular}

Water and lopamidol have been excluded from the table as no nutritional information is provided. All values are given per $100 \mathrm{ml}$ from concentrate. Estimated iron, manganese and copper content of standard juices were obtained from the USDA National Nutrient Database (http://ndb.nal.usda.gov/).

were: field of view (FOV) $25 \times 25 \mathrm{~cm}$, slice thickness $5 \mathrm{~mm}$, matrix $256 \times 256$, TR 7.2, TE $3.3 \mathrm{~ms}$.

The $\mathrm{T}_{2}$ values were measured using an 8-echo spin echo sequence. Eight different echo times (TE) were used, 30, $60,90,120,150,180,210,240 \mathrm{~ms}$. Other imaging parameters were: FOV $25 \times 25 \mathrm{~cm}$, slice thickness $5 \mathrm{~mm}$, matrix $256 \times 256$, TR $2000 \mathrm{~ms}$.

We then measured the signal intensity of each contrast medium using the IR-SSFSE sequences at variable TI times (0-4000 ms), to simulate the in-vivo environment, and subtracted the signal intensity of milk to predict the possible optimal TI times in vivo.

\section{Interactive IR-SSFSE}

Interactive MRI in-vivo was performed using an IRSSFSE pulse sequence. This modified pulse sequence has been custom developed to operate within a proprietary interactive imaging interface (i/Drive Pro Plus; GE Healthcare) which allows interactive control over many imaging parameters such as image plane location, phase encode ordering, and the use of a $180^{\circ}$ inversion pulse that can be interactively toggled on and off prior to the SSFSE readout. The inversion time (TI) between the $180^{\circ}$ inversion pulse and the SSFSE readout could also be interactively controlled during imaging, by inputting different IR times in ms. Initial imaging parameters for the SSFSE sequence were: FOV $25 \times 25 \mathrm{~cm}$, matrix $256 \times 256$, TR $2500 \mathrm{~ms}$, TE $50.1 \mathrm{~ms}$, single slice thickness of $10 \mathrm{~mm}$. No motion suppression or breathing suppression technique was used.

In volunteer imaging, initial localiser images and noninteractive axial and coronal FIESTA and SSFSE sequences

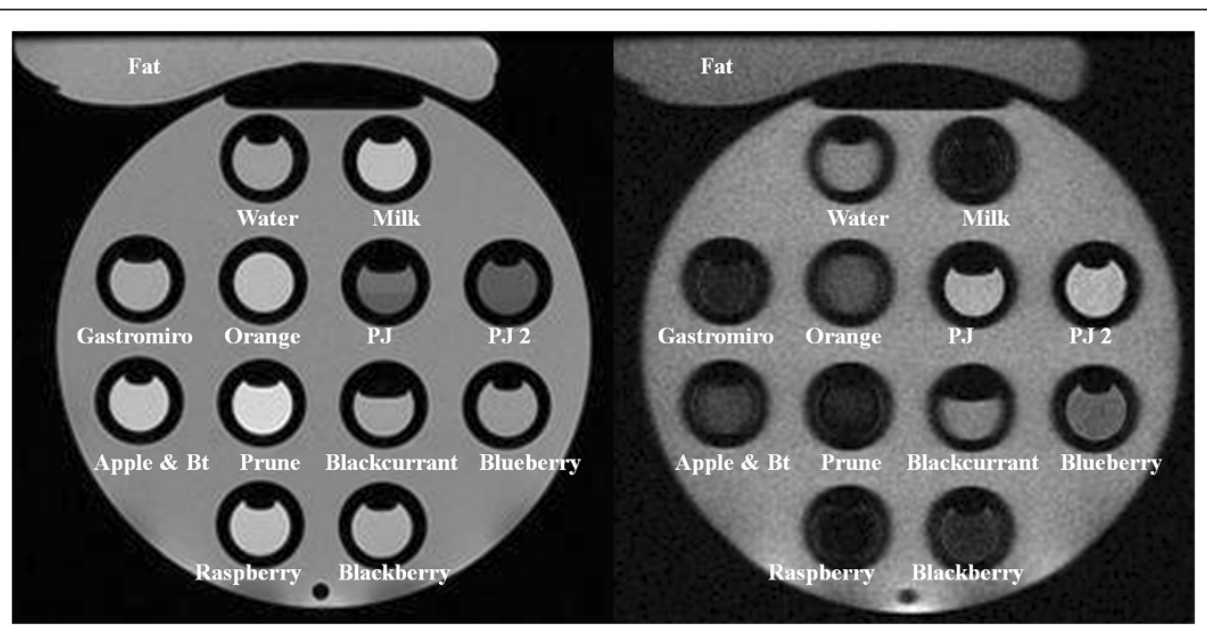

Figure 112 different contrast media assessed in-vitro. Pineapple juice (PJ and PJ2) have the lowest signal on T2W imaging (left : PDW SSFSE; TR 2500), but highest signal recovery on T1w imaging (right: IR-FSPGR with TI =640 ms). 
were obtained in a supine position to outline upper gastrointestinal anatomy and localise pre-existing small bowel contents. Coronal interactive IR-SSFSE was then used during free breathing to vary the TI at $100 \mathrm{~ms}$ intervals (between 0 and $2000 \mathrm{~ms}$ ), and signal intensities were measured in 3 regions of interest (ROIs): stomach, proximal small bowel (duodenum) and mid-small bowel (jejunum; Figure 2), in order to characterise the signal properties of pre-existing bowel contents.

We then repeated the study with increasing TIs within 10 minutes of the oral administration of $200 \mathrm{ml}$ pineapple juice to three adult volunteers, and after $20 \mathrm{ml}$ pineapple juice administration to a single neonatal infant ( 2 week old $3 \mathrm{~kg}$ male infant). We did not use any form of respiratory or cardiac gating.

\section{Results}

In vitro testing

Figure 1 demonstrates the phantom used for in-vitro testing. The $T_{1}$ and $T_{2}$ relaxation times for the contrast media tested are reported in Table 1: pineapple juice from juice (PJ) was found to have both the shortest $\mathrm{T}_{1}(243 \mathrm{~ms})$ and $\mathrm{T}_{2}$ (48 $\left.\mathrm{ms}\right)$ relaxation times.

Assuming that milk was likely to be the main constituent of pre-existing bowel contents in pre-weaned infants, a representative graph of the relative difference in signal intensity between PJ and formula milk is shown in Figure 3, demonstrating that maximal differences are seen in-vitro with TIs between $500-900 \mathrm{~ms}$.

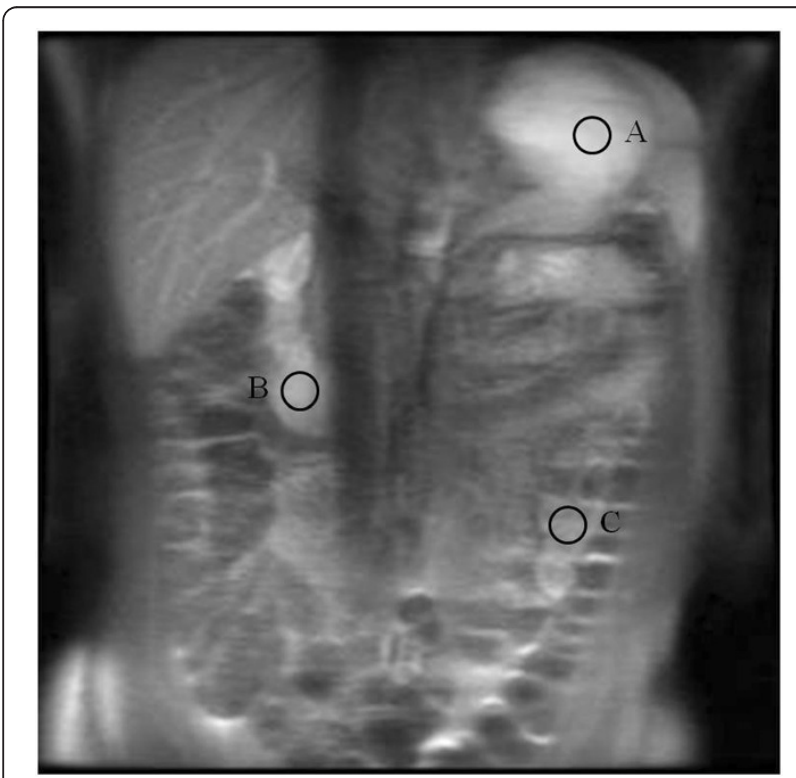

Figure 2 Coronal IR-SSFSE images through the abdomen in an adult following oral pineapple juice administration. Signal intensity was measured in 3 regions of interest: the stomach $(\mathbf{A})$ proximal small bowel (duodenum; B) and mid-small bowel (jejunum; C).

\section{In vivo strategy}

We subsequently tested pineapple juice as an in vivo contrast agent using the IR-SSFSE sequence: we measured signal intensity in three different ROIs (Figures 2), with increasing T1 at $100 \mathrm{~ms}$ intervals $(0-2000 \mathrm{~ms}$; Figure 4) before and after oral PJ administration. By subtracting the signal intensity of distal (jejunal) small bowel contents, we plotted differences in signal intensity (Figure 5) and showed that maximum differences between stomach and jejunum contents were observed at TIs of $1000 \mathrm{~ms}$, $1100 \mathrm{~ms}$, and $900 \mathrm{~ms}$ in the three adult volunteers respectively (Figure 6). We were unable to obtain good images of every TI in the neonate following $20 \mathrm{ml} \mathrm{PJ}$ administration due to patient motion, but maximal signal differences were seen at $1000 \mathrm{~ms}$ (Figure 7).

\section{Discussion}

This study demonstrates that pineapple juice could be used as a positive contrast agent for the proximal neonatal gut. By tailoring an IR-SSFSE sequence to null the signal from existing bowel fluid and thereby optimise the signal from PJ, we have developed a strategy for bowel visualisation using MRI which could be used in a similar fashion to the traditional UGI series in children. The true diagnostic performance of an interactive MRI strategy under these conditions remains to be evaluated.

IR-SSFSE sequences have previously been used for MR imaging of the abdomen [12], but not with interactive control. Our strategy used the IR-SSFSE sequence to effectively suppress bowel fluid signal where its properties were unknown, and to optimally tune the IR timing to provide optimal signal from a contrast agent with known signal properties. Several vendors may offer an IRprepared sequence, such as T1-scout series or modified look-locker inversion recovery (MOLLI) sequences, where changes in inversion recovery time can be used to null myocardium during delayed enhanced imaging, for instance. However they do not allow for on-the-fly adjustment of the TI, which is necessary for rapid optimisation in a paediatric setting. Our strategy helps to tailor the examination to the individual, and has the potential to reduce overall examination time, although this remains to be evaluated in clinical practice.

One of the advantages of using the $T_{1}$ shortening properties of fruit juices are that smaller volume can be used as gut distension is not a requirement. Here, we were able to administer $200 \mathrm{ml}$ to an adult, less than some studies using blueberry juice in adults (400-600 ml) $[9,13,14]$. Using only $20 \mathrm{ml}$ in a neonate is comparable to that used in an UGI and is less than normal meal intakes. The majority of other substances used for MR gut imaging are typically combined with gut-distending agents such as mannitol, known to give gastrointestinal side effects such as flatulence 


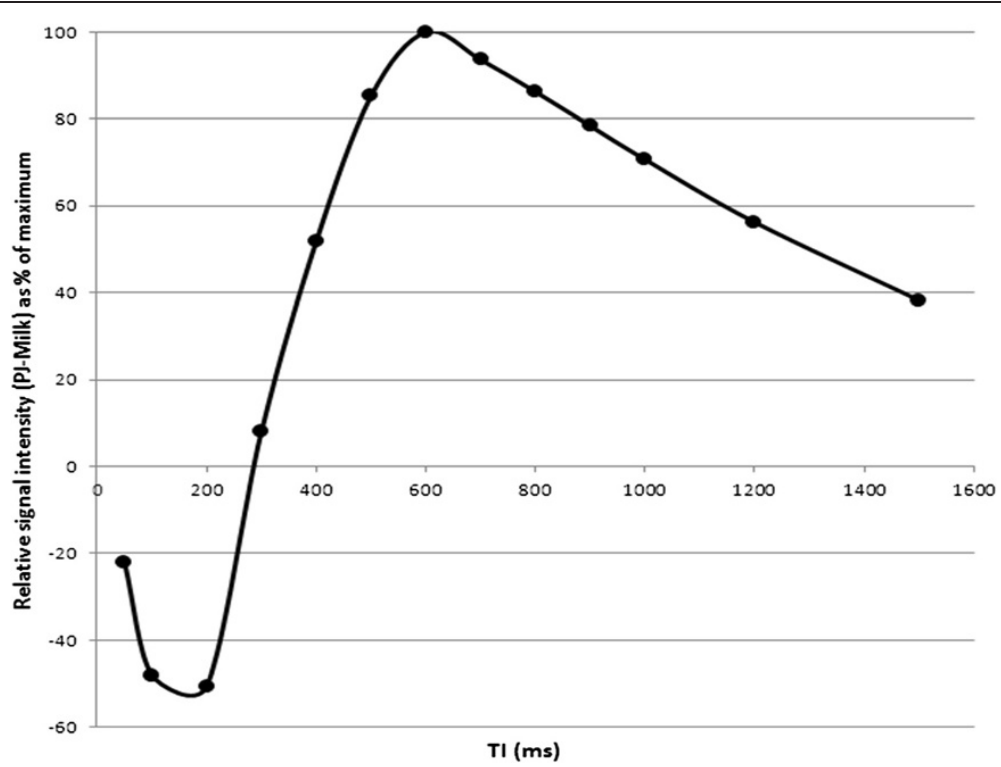

Figure 3 By subtracting the signal intensity of PJ and milk, we were able to plot the relative differences in signal (plotted as a \% of maximum) between the two contrast media at different Tls. Maximal contrast differences are seen with TI between $500-900$ ms.

and bloating, but still widely used for small bowel MR studies in older children and adults.

Despite differences in commercial products and dilutions, our measurements closely mirror those found in other studies, particularly regarding the short $T_{1}$ and $\mathrm{T}_{2}$ of PJ [15]. We included formula milk in our evaluation and obtained similar values to the range previously measured (640-1750 ms) [16]. Several other fruit juices have been proposed as good gut contrast agents, but have only been evaluated in adults, including concentrated blueberry juice and blackberry juice $[13,17,18]$.
The main difference between our phantom study and some previous experiments is likely to relate to the dilutions used; several previous studies measured the signal characteristics of concentrated fruit juices, or indeed fruit pulp itself [17]. Concentrated fruit juices can stimulate gut transit in non-weaned infants, causing diarrhoea and nappy irritation, which can be both uncomfortable and distressing for the child and care-giver, which is why we used diluted products. It is widely reported that the signal suppression effect is due to $\mathrm{T}_{2}$ relaxation caused by their inherently high iron (blackberry) or manganese (pineapple, beet juice, green tea) content, although the
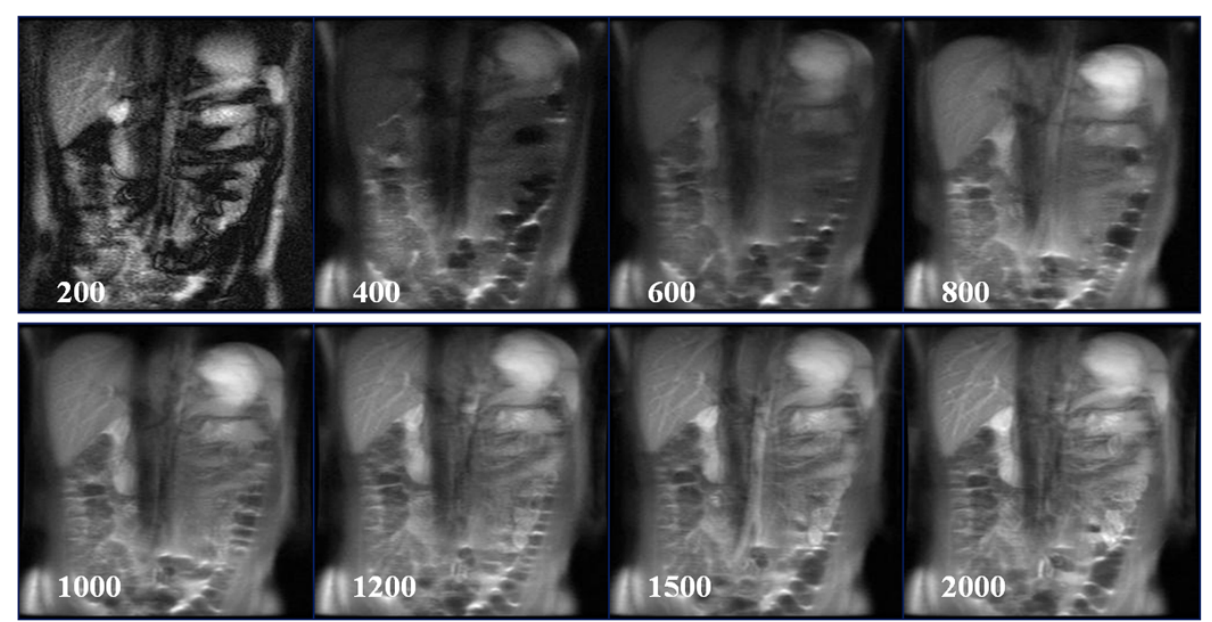

Figure 4 Coronal IR-SSFSE images ( $\mathrm{TI}=1200 \mathrm{~ms}$ ) through the abdomen in an adult following oral pineapple juice administration. Examples of different Tl times (200 - 2000 ms) are given. Relative signal intensity from this study is given in Figure 5 . 


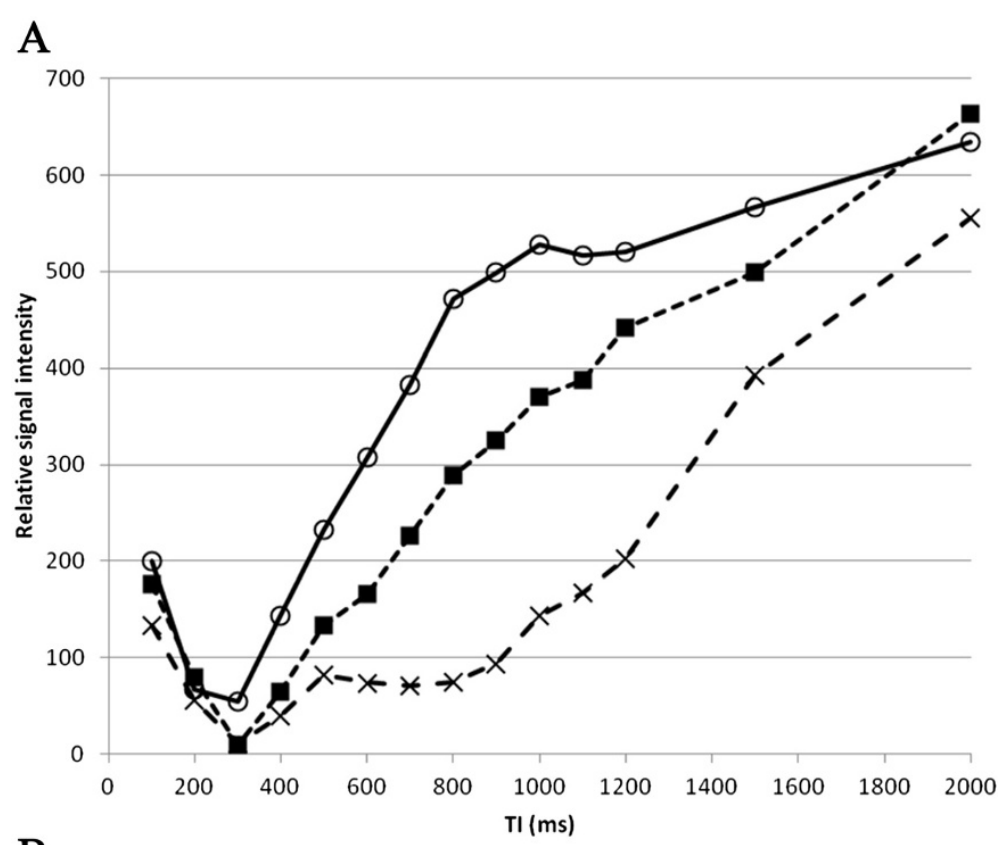

B

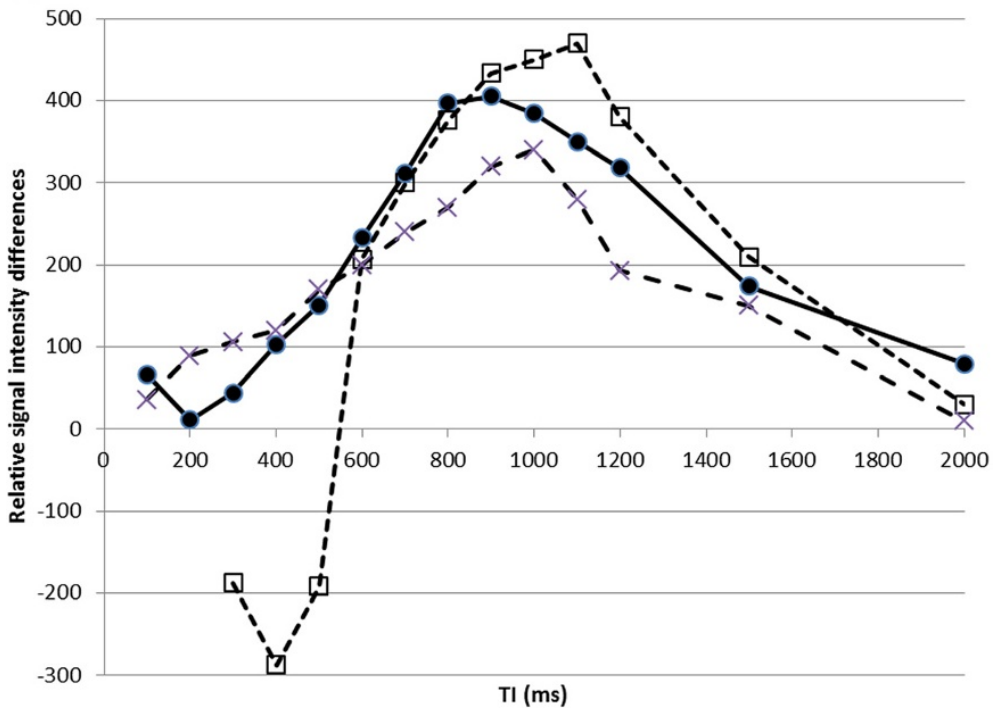

Figure 5 A. Relative signal intensity in three gut regions (A, B and C in Figure 2) following oral pineapple juice administration. ROls were A: stomach (open circles, solid line), B : proximal small bowel (filled squares, dashed line), and C: more distal small bowel (cross, dotted line). B. Relative signal intensity differences (as a \% of maximum) between stomach and distal small bowel (A - C; filled circles, solid line), and between proximal and more distal small bowel ( $B-C$; open squares, dashed line).

exact contents of different commercial fruit juices vary widely, and the precise paramagnetic component (iron, copper or manganese) that gives the relaxation shortening property is debatable. We estimated the paramagnetic agent contents of each of our fruit juices (Table 2) and hypothesise that it may be the manganese content of pineapple juice which gave favourable properties in this study.

Several other oral agents have been developed and have been comprehensively reviewed elsewhere [19]. These can be grouped into predominantly positive gastrointestinal
MR imaging contrast media, typically gadolinium-based compounds e.g. Magnevist Enteral ${ }^{\circ}$ (Schering AG, Berlin, Germany) or Gadolinium zeolite (Gadolite; Pharmacyclics, Sunnyvale, CA, now GlaxoSmithKline Ltd, Brentford, UK), or negative gastrointestinal MR imaging contrast agents, particularly iron-containing solutions such as Perflubron ${ }^{\circ}$ (Imagent $\mathrm{GI}^{\circ}$, Alliance Pharma), or Abdoscan ${ }^{\circ}$ (NycomedAmersham, Oslo, Norway). Most of these are not commercially available, and are not licensed for children. Carbon dioxide gas has also been used as a negative contrast agent 


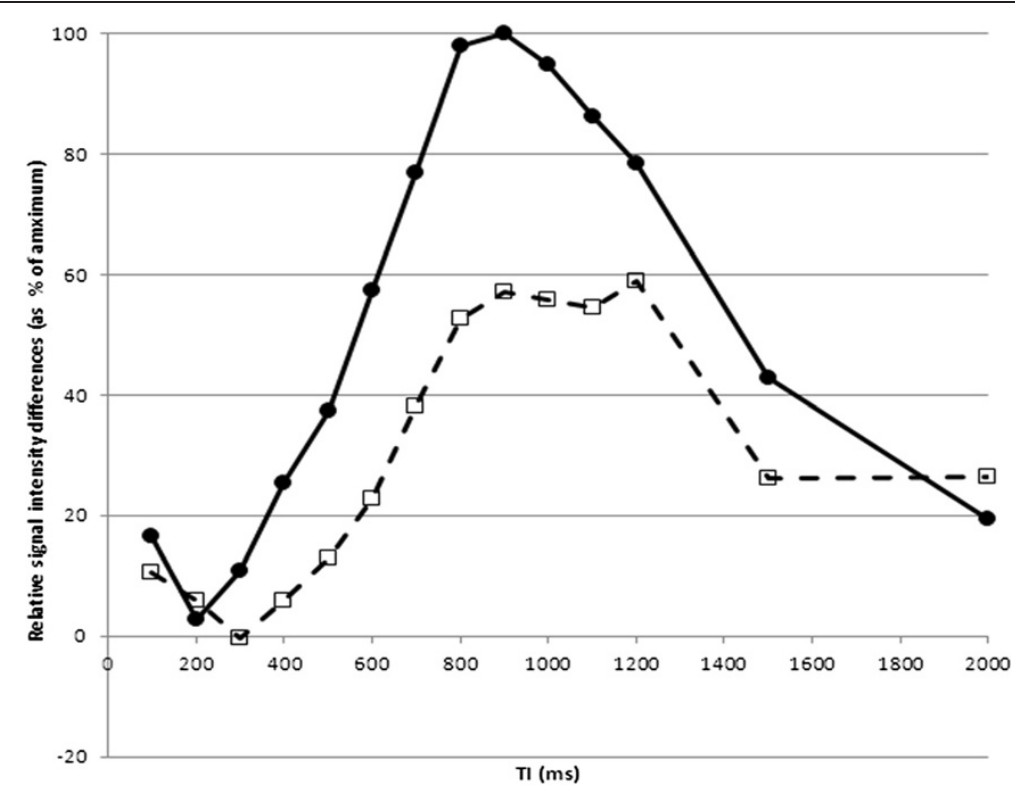

Figure 6 Relative signal intensity differences between stomach and proximal small bowel, with maximum differences observed at Tls of $1000 \mathrm{~ms}, 1100 \mathrm{~ms}$, and $\mathbf{9 0 0} \mathrm{ms}$ in three individual adult volunteers. The shape of each graph was similar, with signal brighter in small bowel than stomach in one individual, which may suggest rapid transit time. The consistent maximum differences at TI of $900-1100 \mathrm{~ms}$ are roughly in keeping with the in-vitro results.

for proximal gut MR imaging and early studies demonstrated acceptable visualisation of the duodenum but were limited in the jejunum and small bowel [20], but this remains to be investigated in neonates.

\section{Limitations of this study}

One limitation of this feasibility study could be the variable content of commercially available fruit juice. We did not determine the exact constituents of the different fruit juices used in this study, but relied on the manufacturers details provided on the packaging and estimated provided by the USDA National Nutrient Database (http://ndb.nal. usda.gov). Since the concentrations of fruit pulp and therefore paramagnetic properties of diluted commercial fruit juices may vary, the ability to interactively optimise the IR timing becomes even more important as a way to compensate for these variations.

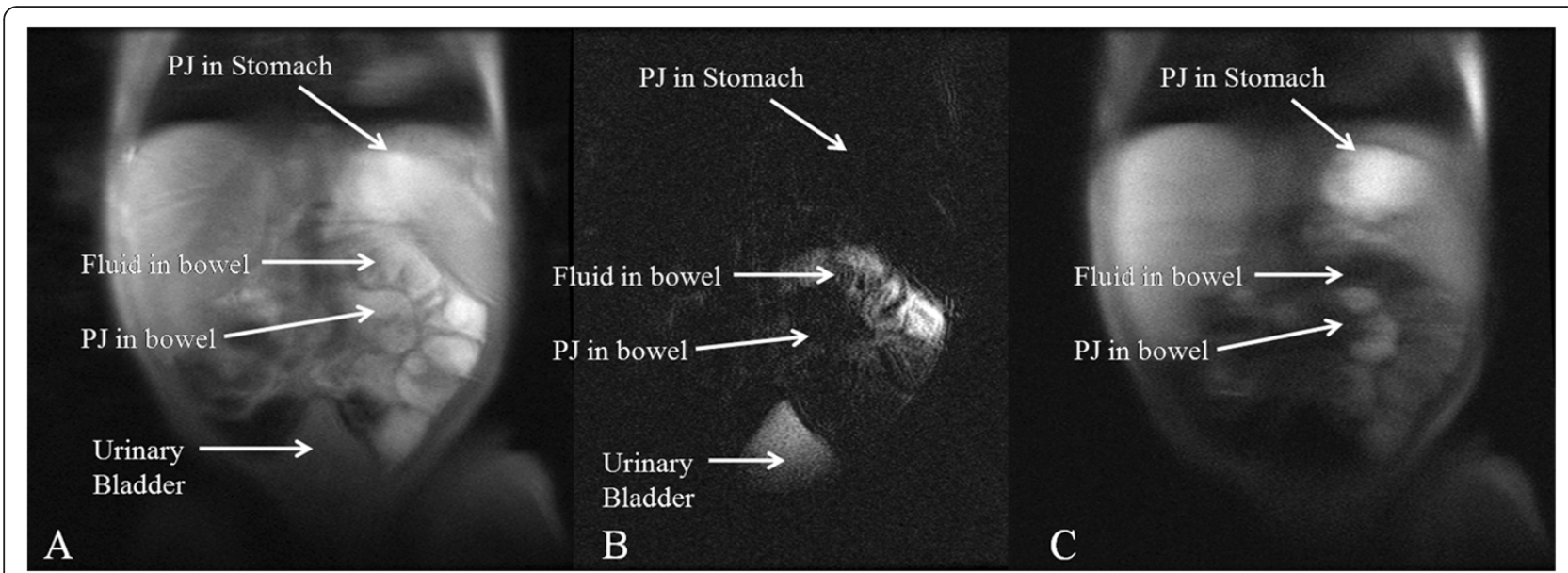

Figure 7 Coronal IR-SSFSE of neonatal GI tract. A 2 week old $3 \mathrm{~kg}$ male infant underwent MRI following PJ administration. A. Conventional SSFSE (TR $2500 \mathrm{~ms}$ ) imaging. It is not possible to differentiate between PJ in the stomach and proximal small bowel from fluid already in the small bowel on conventional T2w imaging. B. T2 $\mathrm{w}$ hydrographic SSFSE image (TR of $4000 \mathrm{~ms}$ ) demonstrating location of pre-existing bowel fluid. The short $T_{2}$ PJ in the stomach and proximal bowel is low signal. C. IR-SSFSE PDW (TI= $1000 \mathrm{~ms}$ ) that nulls the signal from pre-existing bowel fluid, and demonstrates PJ as high signal in stomach and proximal bowel. 
We acknowledge that we did not include any patients in this volunteer feasibility study, and have tested only a limited number of volunteers. The full diagnostic potential of this technique now needs to be evaluated in a larger patient population.

\section{Conclusion}

In conclusion, this work indicates that pineapple juice is the most promising natural commercially available short $\mathrm{T}_{1}$ contrast medium suitable for neonatal GI tract imaging using an interactively adjusted inversion-recovery SSFSE strategy. This allows for pre-existing longer $\mathrm{T}_{1}$, bowel contents to be suppressed, whilst the short $\mathrm{T}_{1}$ of the PJ acts as a positive contrast medium to delineate the gut. This approach allows individual tailoring of the examination, to give optimal discrimination of an orally administered contrast agent from the background signal of gut contents. Further work is required using this strategy in a larger cohort of neonates and infants to evaluate both technical and diagnostic performance.

\section{Competing interests}

The authors declare that they have no competing interests, financial or non-financial

\section{Authors' contributions}

OA, MJG, IJ and ADE carried out the in-vitro studies, and OA, MJG, IJ and PS carried out the in-vivo interactive MRI studies. All authors participated in the design of the study and helped to draft the manuscript. All authors read and approved the final manuscript.

\section{Acknowledgements}

OJA was funded by a Medical Research Council/Royal College of Radiologists Clinical Research Training Fellowship. The views expressed in this publication are those of the author(s). The project was supported by the Addenbrookes Charitable Trust, and the NIHR comprehensive Biomedical Research Centre award to Cambridge University Hospitals NHS Foundation Trust in partnership with the University of Cambridge. None of the funding bodies had any role in analysis of data, results or conclusions of the study.

\section{Author details}

${ }^{1}$ Department of Radiology, Cambridge University Hospitals NHS Foundation Trust, Cambridge CB2 OQQ, UK. ²Department of Radiology, Great Ormond Street Hospital for Children NHS Foundation Trust, London, UK. ${ }^{3} \mathrm{UCL}$ Institute of Child Health, London, UK.

Received: 20 May 2014 Accepted: 21 August 2014

Published: 22 September 2014

\section{References}

1. Long FR, Kramer SS, Markowitz Rl, Taylor GE: Radiographic patterns of intestinal malrotation in children. Radiographics 1996, 16:547-556.

2. Sizemore AW, Rabbani KZ, Ladd A, Applegate KE: Diagnostic performance of the upper gastrointestinal series in the evaluation of children with clinically suspected malrotation. Pediatr Radiol 2008, 38:518-528.

3. Daneman A: Malrotation: the balance of evidence. Pediatr Radiol 2009, 39:S164-S166.

4. Taylor GA: CT appearance of the duodenum and mesenteric vessels in children with normal and abnormal gut rotation. Pediatr Radiol 2011, 41:1378-1383.

5. Graves MJ, Wong P, Black RT, Lomas DJ: MR fluoroscopy employing interactive pulse sequence switching. Proc ISMRM 2007, 15:1654.

6. Makki M, Graves MJ, Lomas DJ: Interactive body MR fluoroscopy using SSFSE RARE with multiparameter control. J MRI 2002, 16:85-93.
7. Arthurs OJ, Edwards AD, Joubert I, Graves MJ, Set PAK, Lomas DJ: Interactive magnetic resonance voiding cystourethrography (iMRVC) for vesicoureteric reflux (VUR) in unsedated infants: a feasibility study. Eur Radiol 2011, 21:1874-1881.

8. Li KCP, Tart RP, Fitzsimmons JR, Storm BL, Mao J, Rolfes RJ: Barium sulfate suspension as a negative oral MRI contrast agent: In vitro and human optimization studies. MRI 1991, 9:141-150.

9. Riordan RD, Khonsari M, Jeffries J, Maskell GF, Cook PG: Pineapple juice as a negative oral contrast agent in magnetic resonance cholangiopancreatography: a preliminary evaluation. $\mathrm{Br} /$ Radiol 2004, 77:991-999.

10. Darge $K$, Anupindi S: Pancreatitis and the role of US, MRCP and ERCP. Pediatr Radiol 2009, 39:5153-7.

11. Baker SS on behalf of the Committee on Nutrition: The use and misuse of fruit juice in paediatrics. Paediatrics 2001, 107:1210-1213.

12. Lauenstein TC, Sharma P, Hughes T, Heberlein K, Tudorascu D, Martin DR: Evaluation of optimized inversion-recovery fat-suppression techniques for T2-weighted abdominal MR imaging. J Magn Reson Imaging 2008, 27:1448-54.

13. Hiraishi K, Narabayashi I, Fujita O, Yamamoto K, Sagami A, Hisada Y, Saika Y, Adachi I, Hasegawa H: Blueberry juice: preliminary evaluation as an oral contrast agent in gastrointestinal MR imaging. Radiology 1995, 194:119-23.

14. Papanikolaou N, Karantanas A, Maris T, Gourtsoyannis N: MR cholangiopancreatography before and after oral blueberry juice administration. J Comput Assist Tomogr 2000, 24:229-234.

15. Asbach P, Breitwieser C, Diederichs G, Eisele S, Kivelitz D, Taupitz M, Zeitz M, Hamm B, Klessen C: Cine magnetic resonance imaging of the small bowel: comparison of different oral contrast media. Acta Radiol 2006, 47:899-906.

16. Balzarini L, Aimeb S, Barberob L, Ceglia E, Petrillo R: Magnetic resonance imaging of the gastrointestinal tract: investigation of baby milk as a low cost contrast medium. Eur Radiol 1992, 15:171-174.

17. Karantanas AH, Papanikolaou N, Kalef-Ezra J, Challa A, Gourtsoyiannis N: Blueberry juice used per os in upper abdominal MR imaging: composition and initial clinical data. Eur Radiol 2000, 10:909-13.

18. Espinosa MG, Sosa M, De León-Rodríguez LM, Córdova T, Bernal-Alvarado J, Avila-Rodríguez M, Reyes-Aguilera JA, Ortíz JJ, Barrios FA: Blackberry (Rubus spp.): a pH-dependent oral contrast medium for gastrointestinal tract images by magnetic resonance imaging. Magn Reson Imaging 2006, 24:195-200.

19. Giovagnoni A, Fabbri A, Maccioni F: Oral contrast agents in MRI of the gastrointestinal tract. Abdom Imaging 2002, 27:367-375.

20. Lomas DJ, Habib SH, Joubert IJ, Sala E, Graves MJ: CO2 as a distending medium for gastric and small bowel MRI: a feasibility study. Eur Radiol 2005, 15:672-6.

doi:10.1186/1471-2342-14-33

Cite this article as: Arthurs et al.: Interactive neonatal gastrointestinal magnetic resonance imaging using fruit juice as an oral contrast media. BMC Medical Imaging 2014 14:33.

\section{Submit your next manuscript to BioMed Central and take full advantage of:}

- Convenient online submission

- Thorough peer review

- No space constraints or color figure charges

- Immediate publication on acceptance

- Inclusion in PubMed, CAS, Scopus and Google Scholar

- Research which is freely available for redistribution 